\title{
Block idempotents and the Brauer correspondence
}

\author{
Jon F. Carlson
}

\begin{abstract}
Let $H$ be a subgroup of a finite group $G$. In this paper the Brauer correspondence between blocks of $H$ and blocks of $G$ is characterized in terms of a relationship among the block idempotents.
\end{abstract}

Let $R$ be an integral domain (commutative with unit element) satisfying the ascending chain condition on ideals. Suppose that $N$, the radical of $R$, is a principal ideal $(N=(\pi))$ and that $R^{*}=R / N$ satisfies the descending chain condition on ideals. Let $N$ contain a rational prime $p=1+1+\ldots+1$. Assume that $R$ is complete with respect to the topology induced by $N$. Then either $\pi=0$ in which case $R=R^{*}$ is a field of characteristic $p$, or $R$ is a complete discrete valuation ring.

Let $G$ be a finite group with a subgroup $H$. Let $R(G)$ denote the group algebra of $G$ with coefficients in $R$. Then $R(H)$ is a subalgebra of $R(G)$. Let $Z(G)$ and $Z(H)$ denote the centers of $R(G)$ and $R(H)$, respectively. If $E$ is a primitive idempotent in $Z(G)$ the block $B=B(E)$ is the collection of all (right) $R(G)$-modules $V$ with $V E=V$. To each primitive idempotent $E$ in $2(G)$ there is associated a linear character $\psi: Z(G) \rightarrow R^{*}$ with $\psi(E)=1^{*}$ and $\psi(E)=0^{*}$ for any primitive idempotent $F \neq E$ in $Z(G)$. We shall assume that $R^{*}$ is a splitting field for $R^{*}(G)$ and for $R^{*}(H)$. Then the linear characters on $Z(G)$ and $Z(H)$ will be in one-to-one correspondence with the primitive idempotents in $Z(G)$ and $Z(H)$ respectively (see [2]). We shall write 
$B \leftrightarrow E \leftrightarrow \psi$ where $B$ is the block of $R(G)$ corresponding to the primitive idempotent in $E$ in $Z(G)$ and $\psi$ is the character of $Z(G)$ associated to $E$.

$$
\begin{array}{r}
\text { For any } \alpha=\sum_{g \in G} a_{g} g \text { in } z(G), \text { let } \\
\theta(\alpha)=\sum_{g \in H} a_{g} g .
\end{array}
$$

Then $\theta: Z(G) \rightarrow Z(H)$ is an $R$-module homomorphism.

DEFINITION. Let $b \leftrightarrow e \leftrightarrow \lambda$ be a block of $R(H)$. If the map $\psi=\lambda \circ \theta: Z(G) \rightarrow R^{*}$ is a linear character of $Z(G)$ corresponding to a block $B \leftrightarrow E \leftrightarrow \psi$ of $R(G)$, then we say that $b^{G}=B$ is defined. The correspondence sending $b$ to $b^{G}$ is called the Brauer correspondence.

Let $\operatorname{rad} Z(H)$ denote the radical of $Z(H)$. We can now state the main result of this paper.

THEOREM. Let $B \leftrightarrow E \leftrightarrow \psi$ and $b \leftrightarrow e \leftrightarrow \lambda$ be blocks of $R(G)$ and $R(H)$ respectively. Then $b^{G}=B$ if and only if $\theta(F) e$ is in the radical of $Z(H)$ for every primitive idempotent $F \neq E$ in $Z(G)$.

Proof. Let $K_{1}, \ldots, K_{s}$ be the conjugate classes of $G$. The ring $Z(G)$ has an $R$-basis consisting of the elements $\hat{K}_{1}, \ldots, \hat{K}_{s}$ where

$$
\hat{K}_{i}=\sum g\left(g \in K_{i}\right) .
$$

In the same way if $L_{1}, \ldots, L_{t}$ are the conjugate classes in $H$ then $\hat{L}_{1}, \ldots, \hat{L}_{t}$ span $Z(H)$. Let $A=A(G: H)$ be the $R$-algebra generated by the class sums $\hat{K}_{1}, \ldots, \hat{K}_{s}$ and $\hat{L}_{1}, \ldots, \hat{L}_{t}$. That is, $A$ is the minimal subalgebra of $R(G)$ which contains both $Z(G)$ and $Z(H)$. The products $\hat{K}_{i} \hat{L}_{j}(i=1, \ldots, 8, j=1, \ldots, t)$ span $A$ as an $R$-algebra.

The map $\theta$ can be easily extended to a map $\theta^{\prime}: A \rightarrow Z(H)$ given by

$$
\theta^{\prime}\left(\hat{K}_{i} \hat{L}_{j}\right)=\theta\left(\hat{K}_{i}\right) \hat{L}_{j}=\left(\sum \hat{L}_{k}\right) \hat{L}_{j} \quad(i=1, \ldots, s ; j=1, \ldots, t),
$$

where the sum is over those $k$ with $L_{k} \subseteq K_{i} \cdot \theta^{\prime}$ is the projection of 
$A$ onto $Z(H)$.

Suppose $b^{G}=B$. Let $F$ be any primitive idempotent in $Z(G)$ with $F \neq E$. If $f \leftrightarrow \phi$ is any primitive idempotent in $Z(H)$ with $f \neq e$ then $f e=0$ and $\phi(\theta(F) e)=0$. Also $\lambda(\theta(F) e)=\lambda \circ \theta(F)=\psi(F)=0$. Since $\theta(F) e$ is in the kernel of every linear character on $Z(H)$ it is in the radical of $Z(H)$.

Conversely suppose that $\theta(F) e$ is in $\operatorname{rad}(H)$ for every primitive idempotent $F$ in $Z(G)$ with $F \neq E$. Let $E=E_{1}, E_{2}, \ldots, E_{m}$ be all of the primitive idempotents in $Z(G)$. Then $E_{1}^{*}, E_{2}^{*}, \ldots, E_{m}^{*}$ are all of the primitive idempotents in $Z^{*}(G)=Z(G) /(\pi) Z(G)$. Since $\sum_{i=1}^{m} E_{i}^{*}=1^{*}$, we have that $e^{*}=\sum_{i=1}^{m} E_{i}^{*} e^{*}$. Now for any $j=1, \ldots, s$,

$$
\hat{K}_{j}^{*} e^{*}=\sum_{i=1}^{m} K_{j}^{*} E_{i}^{*} e^{*}=\sum_{i=1}^{m} \Psi_{i}\left(\hat{K}_{j}\right) E_{i}^{*} e^{*}
$$

where $E_{i} \leftrightarrow \psi_{i}$. Then

$$
\begin{aligned}
\lambda\left(\theta\left(\hat{K}_{j}\right)\right) e^{*} & \equiv \theta\left(\hat{K}_{j}^{*}\right) e^{*} \equiv \theta^{\prime}\left(\hat{K}_{j}^{*} e^{*}\right) \\
& \equiv \psi\left(\hat{K}_{j}\right) \theta^{\prime}\left(E^{*} e^{*}\right) \\
& \equiv \psi\left(\hat{K}_{j}\right) e^{*} \bmod \left(\operatorname{rad} Z^{*}(H)\right),
\end{aligned}
$$

since $e^{*}=\theta^{\prime}\left(e^{*}\right)=\theta^{\prime}\left(\sum E_{i}^{*} e^{*}\right) \equiv \theta^{\prime}\left(E^{*} e^{*}\right) \bmod \left(\operatorname{rad} Z^{*}(H)\right)$. Hence $\lambda \circ \theta=\psi$ on $Z(G)$.

COROLLARY. Let $B \leftrightarrow E \leftrightarrow \psi$ and $b \leftrightarrow e \leftrightarrow \lambda$ be brooks of $R(G)$ and $R(H)$ respectively. If $b^{G}$ is defined then $b^{G}=B$ if and only if $\theta(E) e \equiv e \bmod (\operatorname{radz}(H))$.

This follows directly from the theorem since if $E_{1}, \ldots, E_{s}$ are all of the primitive idempotents in $Z(G)$ then

$$
e=\theta^{\prime}(e)=\theta^{\prime}\left(\sum E_{i} e\right)=\sum \theta\left(E_{i}\right) e \text {. }
$$


REMARK 1. The condition $\theta(E) e \equiv e \bmod (\operatorname{rad}(H))$ is not sufficient to guarantee that $b^{G}$ is defined. For example let $G=\left\langle x|| x^{3}=1\right\rangle$, $H=\{1\}$, and $R=z_{2}[\alpha]$, the algebraic extension of the field with two elements by an element $\alpha$ with $\alpha^{2}+\alpha+1=0$. Then the elements $E_{1}=1+x+x^{2}, E_{2}=1+\alpha x+\alpha^{2} x^{2}$, and $E_{3}=1+\alpha^{2} x+\alpha x^{2}$ are all of the idempotents in $Z(G)$. But $e=1$ is the only idempotent in $Z(H)$ and $\theta\left(E_{1}\right) e=\theta\left(E_{2}\right) e=\theta\left(E_{3}\right) e=e$.

REMARK 2. If $H$ is a normal subgroup of $G$ then $B=b^{G}$ if and only if $E e=e$ (see [4]).

REMARK 3. Using these results it is easy to prove the well known result that if $b^{G}=B$ then any defect group of $b$ is conjugate to a subgroup of some defect group of $B$ (see [1]). For suppose $Z(G: H)$ denotes the centralizer of $H$ in $R(G)$. If $D$ is a defect group of $b$ then by [3] the $R$-subalgebra $Z_{D}(G: H)$, spanned by those class sums of conjugate-in- $H$ elements of $G$ with defect groups conjugate to subgroups of $D$, is an ideal in $Z(G: H)$. Since $\theta(E) e \equiv e \bmod (\operatorname{radz}(H))$ it is clear that some defect group of $B$ must contain $D$.

\section{References}

[1] Richard Brauer, "Zur Darstellungstheorie der Gruppen endlicher Ordnung. II", Math. 2. 72 (1959), 25-46.

[2] Walter Feit, Representations of finite groups, Part I (Mimeographed Notes, Yale University, New Haven, 1969 ).

[3] Masaru Osima, "Notes on blocks of group characters", Math. J. Okayoma Univ. 4 (1955), 175-188.

[4] D.S. Passman, "Blocks and normal subgroups", J. Algebra 12 (1969), 569-575.

Department of Mathematics, University of Georgia, Athens, Georgia, USA. 